\title{
Solving a Linear Programming with Fuzzy Constraint and Objective Coefficients
}

\author{
Hamid Reza Erfanian \\ Department of Mathematics, University of Science and Culture, Tehran, Iran. \\ Corresponding author E-mail: Erfanian@usc.ac.ir \\ Mohammad Javad Abdi and Sahar Kahrizi \\ Department of Mathematics, University of Science and Culture, Tehran, Iran. \\ E-mail: \{abdi.mohammad.javad, sahar.kahrizi92\}@g mail.com
}

\begin{abstract}
In this paper, we consider a method for solving a linear programming problem with fuzzy objective and coefficient matrix, where the fuzzy numbers are supposed to be triangular. By the proposed method, the Decision Maker will have the flexibility of choosing. The solving method is based on the Pareto algorith $\mathrm{m}$, which converts the problem to a weightedobjective linear programming. For more illustration, after discussing the problem and the algorithm, we present an example, which its solutions are independent from the objective weights.
\end{abstract}

Index Terms-Fuzzy Linear Programming, Fuzzy Numbers, Pareto Algorithm, Multi-Objective Linear Programming.

\section{INTRODUCTION}

In the recent years, the optimal decision making is a difficult matter for managers of the big dynamic companies and organizations. For making optimal decisions the managers are facing different constraints such as the limited financial and human resources. One of the most common and simple tools which is used to achieve the best outcome(such as maximizing the profit or minimization the cost) subject to different constraints is the Linear Programming (LP) methods. In general, a linear programming problem can be written as:

$$
\max (\min ) z=c x
$$

subject to

$$
\begin{aligned}
& A x \leq b \\
& x \geq 0
\end{aligned}
$$

In practice, all of the needed information such as $c, A, b$ are not completely available or determined, or in the other sense, these parameters include uncertainty and they are called fuzzy variables. In the recent years, fuzzy logic and optimization have found many applications in different areas. The fuzzy decision making was introduced by Bellman and Zadeh for the first time in
1970 [1], and the in 1974 the mathematical fuzzy programming was proposed by Tanaka et al [16]. Ramik and Rimanek [14] also dealt with an LP problem with fuzzy parameters in the constraints. Later, Delgado et al. [3] studied a general model for fuzzy LP problems which includes fuzziness both in the coefficients and in the accomplishment of the constraints. The first fuzzy linear programming formulation was discussed by Zimmermann in 1978 [20], and then many other models and methods were suggested by others. Negoita developed a formulation for fuzzy linear programming problem based on the fuzzy coefficient matrix [10]. Then, in 2003 multiobjective fuzzy linear programming problem was established by Zhang et al [18]. In the last years, Erfan ian et al. have worked on some concepts of generalized derivative of fuzzy nonsmooth functions, generalized first and second derivative for nonsmooth functions and nonsmooth optimal control[4,5,11,12]

In this paper, linear programming problems with the fuzzy resources and coefficients are discussed. Hence, in section 2, some of the basic definitions and concepts of fuzzy sets and fuzzy optimization are reviewed. In section 3 , the concepts of ranking functions are introduced. In section 4, a fuzzy linear programming with fuzzy resources and coefficients is considered, and in section 5, multi-objective fuzzy linear programs are discussed. Finally, in section 6, a practical example is solved and in the last section the results are summarized.

\section{PRELIMINARIES}

In this section, we review some of the definitions and concept of the fuzzy sets and calculus [19].

A.--

Suppose $X$ is an arbitrary universal set. Then a fuzzy subset $\tilde{A}$ in $X$ is defined as follows:

$$
\tilde{A}=\left\{\left(x, \mu_{\tilde{A}}(x)\right) \mid x \in X\right\}
$$

where $\mu_{\tilde{A}}(x): X \rightarrow[0,1]$ is the so called membership function. 
B.--

A fuzzy set $\tilde{A}$ is normal if there exists $x_{0} \in X$ with $\mu_{\tilde{A}}\left(x_{0}\right)=1$

C.--

The support of a fuzzy set A is a set which its members have non-zero membership degree, and is defined as follows:

$$
\operatorname{Supp}(A)=\left\{x \in X \mid \mu_{A}(x)>0\right\}
$$

D.--

A weak cut of a fuzzy set is a set which its members belong to the universal set and its membership function in the fuzzy set $\tilde{A}$ has the value equal or greater than $\alpha$.

$$
A_{\alpha}=[A]^{\tilde{\alpha}}=\left\{x \in X \mid \mu_{A}(x) \geq \alpha, \alpha \in[0,1]\right\}
$$

In the definition above, if one neglects the equality condition of the membership degree, then the achieved set is called a strong $\alpha$-cut.

E.--

A fuzzy set $A$ in $X=R^{n}$ is convex if every $\alpha$-cut of $A$ for any $\alpha(0<\alpha \leq 1)$ is convex. In other words, a fuzzy set $A$ is convex if and only if for every $x_{1}, x_{2} \in X$ and $\lambda \in[0,1]$ the following inequality holds:

$$
\mu_{\tilde{A}}\left[\lambda x_{1}+(1-\lambda) x_{2}\right] \geq \min \left[\mu_{\tilde{A}}\left(x_{1}\right), \mu_{\tilde{A}}\left(x_{2}\right)\right]
$$

\section{F.--}

A fuzzy set $A$ on real numbers $R$, which satisfies the following condition is called a fuzzy number.

1- $A$ is a convex set.

2- There exist only one unique $x \in R$, such that $\mu_{A}(x)=1$.

3- $\mu_{A}(x)$ is piecewise continuous.

\section{G.--}

A triangular fuzzy number with the center $S$ the left bound $l>0$, and the right bound $r>0$ has the following membership function:

$$
\mu_{A}(x)=\left\{\begin{array}{lc}
\frac{x-(s-l)}{l}, & s-l<x<s \\
\frac{(s+l)-x}{r}, & s<x<s+l \\
0, & \text { o.w. }
\end{array}\right.
$$

H.--

Suppose the triangular fuzzy numbers $A$ and $B$ are $\left(a_{1}, a_{2}, a_{3}\right)$ and $\left(b_{1}, b_{2}, b_{3}\right)$ respectively $a_{2}, b_{2}$ are the centers $a_{3}, b_{3}$ are the biggest and $a_{1}, b_{1}$ are the smallest possible values. Then the addition and subtraction of these two numbers are defined as follows:

$$
\begin{aligned}
A \oplus B & =\left(a_{1}, a_{2}, a_{3}\right) \oplus\left(b_{1}, b_{2}, b_{3}\right) \\
& =\left(a_{1}+b_{1}, a_{2}+b_{2}, a_{3}+b_{3}\right) \\
A \ominus B & =\left(a_{1}, a_{2}, a_{3}\right) \ominus\left(b_{1}, b_{2}, b_{3}\right) \\
& =\left(a_{1}-b_{3}, a_{2}-b_{2}, a_{3}-b_{1}\right)
\end{aligned}
$$

I.--

If $A$ and $B$ are the triangular fuzzy numbers in the form of $A=(a, b, c), B=(x, y, z)$, then we have [7]:

$$
A \otimes B= \begin{cases}(a x, b y, c z), & a \geq 0 \\ (a z, b y, c z), & a<0, c \geq 0 \\ (a z, b y, c x), & c<0\end{cases}
$$

\section{RANKING OF FUZZY NUMBERS}

In this section, we present a new approach to fuzzy ordinary where for any two triangular fuzzy numbers $\tilde{a}=\left(s_{a}, l_{a}, r_{a}\right)$ and $\tilde{b}=\left(s_{b}, l_{b}, r_{b}\right), \tilde{a} \prec \tilde{b}$ if and only if

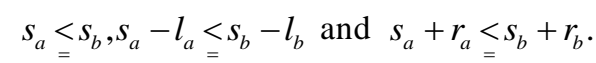

J.--

Assume $\tilde{a}=\left(s_{a}, l_{a}, r_{a}\right)$ and $\tilde{b}=\left(s_{b}, l_{b}, r_{b}\right)$ to be two triangular fuzzy numbers. The definitions of the relations ' $\approx$ ' and ' $\prec$ ' are given in below:

$$
\begin{aligned}
& \tilde{a} \approx \tilde{b} \text { iff } s_{a}=s_{b}, s_{a}-l_{a}=s_{b}-l_{b}, s_{a}+r_{a}=s_{b}+r_{b}, \\
& \tilde{a} \prec \tilde{b} \text { iff } s_{a}<s_{b}, s_{a}-l_{a}<s_{b}-l_{b}, s_{a}+r_{a}<s_{b}+r_{b} .
\end{aligned}
$$

Remark 1: Denote $\tilde{a} \prec \tilde{b}$ if and only if $\tilde{a} \approx \tilde{b}$ or $\tilde{a} \prec \tilde{b}$ and let $\tilde{0}=(0,0,0)$ be a zero triangular fuzzy number. Thus, any $\tilde{a}$ such that $\tilde{a} \approx \tilde{0}$, is a zero too.

Lemma 1: Assume $\tilde{a} \prec \tilde{b}$ Than $-\tilde{a} \succ-\tilde{b}$.

Proof. It is straightforward.

Lemma 2: Assume $\tilde{a}, \tilde{b}, \tilde{c} \in F(\mathbb{R})$. Than,

1) $\tilde{a} \approx \tilde{a}$ for every $\tilde{a}$ (reflexivity);

2) $\quad \tilde{a} \approx \tilde{b}$,than $\tilde{b} \approx \tilde{a}$ (symmetry);

3) $\tilde{a} \approx \tilde{b}$ and $\tilde{b} \approx \tilde{c}$, than $\tilde{a} \approx \tilde{c}$ (transitivity). 
Proof. It is straightforward.

Remark 2: In fact, Lemma up shows that the relation ' $\approx$ ' is an equivalence relation on $F(\mathbb{R})$. Moreover, if $\tilde{a}$ is an element of $F(\mathbb{R})$, the fuzzy subset of $F(\mathbb{R})$ defined by

$$
[\tilde{a}]=\{\tilde{b} \in F(\mathbb{R}) \mid \tilde{a} \approx \tilde{b}\}
$$

is called the equivalence fuzzy set of $\tilde{a}$ is thus the set of all elements which are equivalent to $\tilde{a}$.

Lemma 3: Assume $\tilde{a}, \tilde{b}, \tilde{c} \in F(\mathbb{R})$. The relation ' $\prec$ ' is a partial order on $F(\mathbb{R})$.

Proof. It is straightforward by evaluation the triple properties: reflexivity, symmetry and transitivity.

Remark 3: We emphasize that the relation ' $\prec$ is a linear order on $F(\mathbb{R})$.

Lemma 4: If $\underset{=}{\tilde{a} \prec \tilde{b}}$ and $\underset{c}{\tilde{c} \prec} \tilde{d}$, then $\tilde{a}+\underset{c}{\tilde{c} \prec} \tilde{b}+\tilde{d}$.

Proof. It is straightforward.

\section{LINEAR PROGRAMMING PROBLEM WITH FUZZY RESOURCES AND COEFFICIENTS}

Consider the following fuzzy linear programming problem $[2,6,9,15]$ :

$$
\begin{gathered}
\langle\tilde{c}, x\rangle=f_{i}\left(x_{j}\right)=f_{i}(x)=\max \tilde{z}=\sum_{j=1}^{n} \tilde{c}_{j} x_{j} \\
\text { subject to } \quad \sum_{j=1}^{n} \tilde{A}_{i j} x_{j} \leq \tilde{B}_{i} \quad(1 \leq i \leq m) \\
x_{j} \geq 0 \quad(1 \leq j \leq n)
\end{gathered}
$$

where $\tilde{A}_{i j}, \tilde{B}_{i}, \tilde{c}_{i}$ are fuzzy numbers. In this case, we assume that all of fuzzy numbers are triangular. As shown in Figure (1), ever triangular fuzzy number can be cast as 3 real numbers $s, l, r$, i.e. $\tilde{A}=(s, l, r)$.

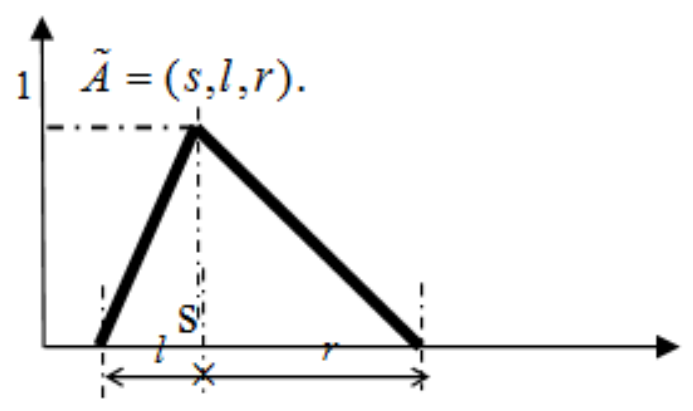

Fig.1. The Fuzzy Number $\tilde{A}$
Now, the model (1) can be written as

$$
\begin{gathered}
\max \langle\tilde{c}, x\rangle=\sum_{j=1}^{n} \tilde{c}_{j} x_{j} \\
\text { s.t. } \quad \sum_{j=1}^{n}\left(s_{i j}, l_{i j}, r_{i j}\right) x_{j} \leq\left(t_{i}, u_{i}, v_{i}\right) \quad(1 \leq i \leq m) \\
x_{j} \geq 0 \quad(1 \leq j \leq n)
\end{gathered}
$$

Theorem 1: For every two fuzzy numbers $A=\left(s_{1}, l_{1}, r_{1}\right), B=\left(s_{2}, l_{2}, r_{2}\right)$ we have $A \leq B$ if and only if $s_{1} \leq s_{2}, s_{1}-l_{1} \leq s_{2}-l_{2}, s_{1}+r_{1} \leq s_{2}+r_{2}$.

Proof. It is straightforward.

Using the above expressions, the model (12) can be converted to a classic linear program as follows:

$$
\begin{gathered}
\max \quad\langle\tilde{c}, x\rangle=\sum_{j=1}^{n} \tilde{c}_{j} x_{j} \\
\text { s.t. } \quad \sum_{j=1}^{n} s_{i j} x_{j} \leq t_{i} \\
\sum_{j=1}^{n}\left(s_{i j}-l_{i j}\right) x_{j} \leq t_{i}-u_{i} \quad(1 \leq i \leq m) \\
\sum_{j=1}^{n}\left(s_{i j}+r_{i j}\right) x_{j} \leq t_{i}+v_{i} \\
x_{j} \geq 0 \quad \quad(1 \leq j \leq n)
\end{gathered}
$$

However, since all nu mbers involved in constraints are real numbers and all goal coefficients are fuzzy numbers, this problem is an essentially fuzzy LP problem.

K.--

A point $x^{*} \in X$ is said to be an optimal solution to the fuzzy LP problem if it holds that (in maximization case) if $\left\langle\tilde{c}, x^{*}\right\rangle \geq\langle\tilde{c}, x\rangle$ for all $x \in X$.

L.--

A point $x^{*} \in X$ is said to be a no dominated solution to the fuzzy LP problem if there does not exist $x^{*} \in X$ such that $\langle\tilde{c}, x\rangle \geq\left\langle\tilde{c}, x^{*}\right\rangle$ holds.

\section{Multi-OBJJECTIVE FUZZY LINEAR PROGRAMMING PROBLEMS}

We consider the following Multi-Objective Linear program (MOLP), which is strongly related to the fuzzy linear programing problem $[9,15]$.

$$
(M O L P)\left\{\begin{array}{lr}
\max & \left(\left\langle c_{0}^{l}, x\right\rangle,\left\langle c_{0}^{c}, x\right\rangle,\left\langle c_{0}^{u}, x\right\rangle\right)^{T} \\
\text { s.t. } & D x \leq d, \quad x \geq 0
\end{array}\right.
$$


where

$$
\begin{aligned}
c_{0}^{l} & =\left(c_{01}^{l}, c_{02}^{l}, \ldots, c_{0 n}^{l}\right)^{T}, \\
c_{1}^{c} & =\left(c_{11}^{c}, c_{12}^{c}, \ldots, c_{1 n}^{c}\right)^{T}, \\
c_{0}^{u} & =\left(c_{01}^{u}, c_{02}^{u}, \ldots, c_{0 n}^{u}\right)^{T} \in \mathbb{R}^{n},
\end{aligned}
$$

and the constraint $D x \leq d$ is constructed using the model (13).

M.--

A point $x^{*} \in X$ is said to be a complete optimal solution to the (MOLP) problem if it holds that $\left(\left(c_{0}^{l}, x^{*}\right),\left(c_{1}^{c}, x^{*}\right),\left(c_{0}^{u}, x^{*}\right)\right)^{T} \geq\left(\left(c_{0}^{l}, x\right),\left(c_{1}^{c}, x\right),\left(c_{0}^{u}, x\right)\right)^{T}$ for all $x \in X$.

N.--

A point $x^{*} \in X$ is said to be a Pareto optimal solution to the (MOLP) problem if there is no $x \in X$, such that $\left(\left(c_{0}^{l}, x^{*}\right),\left(c_{1}^{c}, x^{*}\right),\left(c_{0}^{u}, x^{*}\right)\right)^{T} \leq\left(\left(c_{0}^{l}, x\right),\left(c_{1}^{c}, x\right),\left(c_{0}^{u}, x\right)\right)^{T}$ holds.

Theorem 2: Let point $x^{*} \in X$ be a feasible solution to the fuzzy LP problem. $\tilde{c}_{1}, \tilde{c}_{2}, \ldots, \tilde{c}_{n}$ are determined by the reference functions, i.e., for the triangular fuzzy number $\tilde{c}_{j}, L_{j}$ and $R_{j}$ are respectively the left and right reference functions, and also $\lambda$ level set of $\tilde{c}_{j}$ is represent as interval $\left[c_{i \lambda}^{L}, c_{i \lambda}^{R}\right]$.If

$$
\begin{aligned}
& L_{1}\left(c_{10}^{L}+(1-\lambda) c_{11}^{m}\right)=L_{2}\left(\lambda c_{10}^{L}+(1-\lambda) c_{21}^{m}\right)=\ldots \\
& =L_{n}\left(\lambda c_{n 0}^{L}+(1-\lambda) c_{n 1}^{m}\right), \\
& R_{1}\left(c_{10}^{L}+(1-\lambda) c_{11}^{m}\right)=R_{2}\left(\lambda c_{10}^{L}+(1-\lambda) c_{21}^{m}\right)=\ldots \\
& =R_{n}\left(\lambda c_{n 0}^{L}+(1-\lambda) c_{n 1}^{m}\right),
\end{aligned}
$$

then $x^{*}$ is an optimal solution to the problem if and only if $x^{*}$ is a complete optimal solution to the (MOLP) problem.

Proof. If $x^{*}$ is an optimal solution to the fuzzy LP problem, then for any $x \in X$, we have $\left\langle\tilde{c}, x^{*}\right\rangle \geq\langle\tilde{c}, x\rangle$. Therefore, for any $\lambda \in[0,1]$,

$$
\begin{aligned}
& \left(\sum_{i=1}^{n} \tilde{c}_{i} x_{i}^{*}\right)_{\lambda}^{L} \geq\left(\sum_{i=1}^{n} \tilde{c}_{i} x_{i}\right)_{\lambda}^{L}, \\
& \left(\sum_{i=1}^{n} \tilde{c}_{i} x_{i}^{*}\right)_{\lambda}^{R} \geq\left(\sum_{i=1}^{n} \tilde{c}_{i} x_{i}\right)_{\lambda}^{R}
\end{aligned}
$$

(if $\lambda_{1}=1$, it is clear that $c_{i 1}^{L}=c_{i 1}^{m}$.)

So

$$
\sum_{i=1}^{n} c_{i \lambda_{1}}^{L} x_{i}^{*} \geq \sum_{i=1}^{n} c_{i \lambda_{1}}^{L} x_{i} .
$$

Similarly,

$\sum_{i=1}^{n}\left(\lambda c_{i 0}^{R}+(1-\lambda) c_{i 1}^{m}\right) x_{i}^{*} \geq \sum_{i=1}^{n}\left(\lambda c_{i 0}^{R}+(1-\lambda) c_{i 1}^{m}\right) x_{i}, \forall \lambda \in[0,1]$

According to Eq. (16), we have

$$
\exists \lambda_{2} \in[0,1], \lambda c_{i 0}^{R}+(1-\lambda) c_{i 1}^{m}=c_{i \lambda_{2}}^{R}, i=1,2, \ldots, n .
$$

(if $\lambda_{2}=1$, it is clear that $c_{i 1}^{R}=c_{i 1}^{m}$.)

So

$$
\sum_{i=1}^{n} c_{i \lambda_{2}}^{R} x_{i}^{*} \geq \sum_{i=1}^{n} c_{i \lambda_{2}}^{R} x_{i}
$$


For fuzzy numbers $\left(\tilde{c}_{i}\right)_{L_{i} R_{i}}, i=1,2, \ldots, n$, since the reference functions $L_{i}$ and $R_{i}$ are strictly decreasing, so $\lambda_{1}$ and $\lambda_{2}$ can take every value in the interval $[0,1]$.We can take the proper $\lambda$ to $\lambda_{1}=\lambda_{2}=\lambda$ as well as suggested also in [6]. Then, we have

$$
\sum_{i=1}^{n} c_{i \lambda}^{L} x_{i}^{*} \geq \sum_{i=1}^{n} c_{i \lambda}^{L} x_{i}
$$

and

$$
\begin{gathered}
\sum_{i=1}^{n} c_{i \lambda}^{R} x_{i}^{*} \geq \sum_{i=1}^{n} c_{i \lambda}^{R} x_{i}, \quad \forall \lambda \in[0,1], \\
\sum_{i=1}^{n} c_{i 1}^{m} x_{i}^{*} \geq \sum_{i=1}^{n} c_{i 1}^{m} x_{i} \quad \text { for } \lambda=1,
\end{gathered}
$$

for any $\lambda \in[0,1]$. Therefore, $x^{*}$ is an optimal solution to the fuzzy LP problem and the proof is completed.

Remark 4: For the fuzzy LP problem, if the fuzzy coefficients $\tilde{c}_{i}, i=1,2, \ldots, n$, have the same shapes, the eqations (15) and (16) hold.

Theorem 3: Let point $x^{*} \in X$ be a feasible solution to the fuzzy LP problem. Then $x^{*}$ is a no dominated solution to the problem if and only if $x^{*}$ is a Pareto optimal solution to the (MOLP) problem.

Proof. Let $x^{*} \in X$ be a no dominated solution to the fuzzy LP problem. On the contrary, we suppose that there exists an $\bar{x} \in X$ such that

$$
\left(\left\langle c_{0}^{L}, x^{*}\right\rangle,\left\langle c_{1}^{m}, x^{*}\right\rangle,\left\langle c_{0}^{R}, x^{*}\right\rangle\right)^{T} \geq\left(\left\langle c_{0}^{L}, \bar{x}\right\rangle,\left\langle c_{1}^{m}, \bar{x}\right\rangle,\left\langle c_{0}^{R}, \bar{x}\right\rangle\right)^{T},
$$

i.e.,

$$
\sum_{i=1}^{n} c_{i 0}^{L} x_{i}^{*} \geq \sum_{i=1}^{n} c_{i 0}^{L} \bar{x}_{i}, \sum_{i=1}^{n} c_{i 1}^{m} x_{i}^{*} \geq \sum_{i=1}^{n} c_{i 1}^{m} \bar{x}_{i}
$$

and

$$
\sum_{i=1}^{n} c_{i 0}^{R} x_{i}^{*} \geq \sum_{i=1}^{n} c_{i 0}^{R} \bar{x}_{i} .
$$

Then

$$
\sum_{i=1}^{n}\left(\lambda c_{i 0}^{L}+(1-\lambda) c_{i 1}^{m}\right) x_{i}^{*} \geq \sum_{i=1}^{n}\left(\lambda c_{i 0}^{L}+(1-\lambda) c_{i 1}^{m}\right) \bar{x}_{i}, \forall \lambda \in[0,1]
$$

According to Eq.(15), we have

$$
\exists \lambda_{1} \in[0,1], \lambda c_{i 0}^{L}+(1-\lambda) c_{i 1}^{m}=c_{i \lambda_{1}}^{L}, i=1,2, \ldots, n .
$$

(if $\lambda_{1}=1$, it is clear that $c_{i 1}^{L}=c_{i 1}^{m}$.)

So

$$
\sum_{i=1}^{n} c_{i \lambda_{1}}^{L} x_{i}^{*} \geq \sum_{i=1}^{n} c_{i \lambda_{1}}^{L} \bar{x}_{i}
$$

Similarly,

$$
\sum_{i=1}^{n}\left(\lambda c_{i 0}^{R}+(1-\lambda) c_{i 1}^{m}\right) x_{i}^{*} \geq \sum_{i=1}^{n}\left(\lambda c_{i 0}^{R}+(1-\lambda) c_{i 1}^{m}\right) \bar{x}_{i}, \forall \lambda \in[0,1]
$$

According to Eq. (16), we have

$$
\exists \lambda_{2} \in[0,1], \lambda c_{i 0}^{R}+(1-\lambda) c_{i 1}^{m}=c_{i \lambda_{2}}^{R}, i=1,2, \ldots, n .
$$

(if $\lambda_{2}=1$, it is clear that $c_{i 1}^{R}=c_{i 1}^{m}$.)

So

$$
\sum_{i=1}^{n} c_{i \lambda_{2}}^{R} x_{i}^{*} \geq \sum_{i=1}^{n} c_{i \lambda_{2}}^{R} \bar{x}_{i}
$$

And for $\lambda_{1}=\lambda_{2}=1$, we have

$$
\sum_{i=1}^{n} c_{i \lambda_{2}}^{m} x_{i}^{*} \geq \sum_{i=1}^{n} c_{i \lambda_{2}}^{m} \bar{x}_{i}
$$

That is $\langle\tilde{c}, \bar{x}\rangle \succ\left\langle\tilde{c}, x^{*}\right\rangle$.

However, this contradicts the assumption that $x^{*} \in X$ is a no dominated solution to the fuzzy LP problem.

On the other side, let $x^{*} \in X$ be a Pareto optimal solution to the (MOLP) problem.

If $x^{*}$ is not a no dominated solution to the problem, then there exists $\bar{x} \in X$ such that $\langle\tilde{c}, \bar{x}\rangle \succ\left\langle\tilde{c}, x^{*}\right\rangle$. Therefore, for any $\lambda \in[0,1]$, we have

$$
\begin{aligned}
& \left(\sum_{i=1}^{n} \tilde{c}_{i} x_{i}^{*}\right)_{\lambda}^{R} \leq\left(\sum_{i=1}^{n} \tilde{c}_{i} \bar{x}_{i}\right)_{\lambda}^{R}, \\
& \left(\sum_{i=1}^{n} \tilde{c}_{i} x_{i}^{*}\right)_{\lambda}^{L} \leq\left(\sum_{i=1}^{n} \tilde{c}_{i} \bar{x}_{i}\right)_{\lambda}^{L},
\end{aligned}
$$

and

$$
\left(\sum_{i=1}^{n} \tilde{c}_{i} x_{i}^{*}\right)_{\lambda}^{m} \leq\left(\sum_{i=1}^{n} \tilde{c}_{i} \bar{x}_{i}\right)_{\lambda}^{m} .
$$

That is

$$
\left\langle c_{\lambda}^{L}, x^{*}\right\rangle \leq\left\langle c_{\lambda}^{L}, \bar{x}\right\rangle,\left\langle c_{\lambda}^{R}, x^{*}\right\rangle \leq\left\langle c_{\lambda}^{R}, \bar{x}\right\rangle
$$

and 


$$
\left\langle c_{\lambda}^{m}, x^{*}\right\rangle \leq\left\langle c_{\lambda}^{m}, \bar{x}\right\rangle
$$

Hence, for $\lambda=0$ and $\lambda=1$, we have

$$
\left\langle c_{0}^{L}, \bar{x}\right\rangle \geq\left\langle c_{0}^{L}, x^{*}\right\rangle,\left\langle c_{1}^{m}, \bar{x}\right\rangle \geq\left\langle c_{1}^{m}, x^{*}\right\rangle,\left\langle c_{0}^{R}, \bar{x}\right\rangle \geq\left\langle c_{0}^{R}, x^{*}\right\rangle .
$$

It contradicts the assumption that $x^{*} \in X$ is a Pareto optimal solution to the (MOLP) problem.

Consider a multiple objective optimization problem with $k$ fuzzy goals $\tilde{z}_{1}, \tilde{z}_{2}, \ldots, \tilde{z}_{k}$, which are introduced by fuzzy sets $\tilde{Z}_{i}, i=1,2, \ldots, k$, and fuzzy constraints are $\tilde{d}_{1}, \tilde{d}_{2}, \ldots, \tilde{d}_{m}$, which are shown by fuzzy sets $\tilde{D}_{j}, j=1, \ldots, m$. By generalizing the analogy from the single objective function, the resulting fuzzy decision is given as

$$
\tilde{Z}_{1} \cap \tilde{Z}_{2} \cap \ldots \cap \tilde{Z}_{k} \cap \tilde{D}_{1} \cap \tilde{D}_{2} \cap \ldots \cap \tilde{D}_{m} .
$$

In terms of corresponding membership values for the fuzzy goals and the fuzzy constraints, the resulting decision is

$$
\mu_{\tilde{f}}(X)=\min \left[\mu_{\tilde{z}_{i}}, \mu_{\tilde{D}_{j}}\right]
$$

An optimum solution $X^{*}$ is one at which the membership function of the resulting decision $\tilde{f}$ is maximu $m$. That is

$$
\mu_{\tilde{f}}\left(X^{*}\right)=\max \mu_{\tilde{f}}(X)
$$

The shape of the membership functions such as a linear, concave, or convex function, for various objectives and constraints, can affect the optimu m solution significantly. A linear approximation has been most commonly used because of simplicity and expediency [17].

Applying the triangular fuzzy parameters as triangular fuzzy number the MOLP will be written as follows:

$$
\begin{aligned}
& \max \tilde{z_{i}}=\sum_{j=1}^{n}\left(c^{c}, c^{l}, c^{u}\right)_{i j} x_{j} \\
& \text { s.t. } \quad \sum_{j=1}^{n} s_{i j} x_{j} \leq t_{i} \\
& \sum_{j=1}^{n}\left(s_{i j}-l_{i j}\right) x_{j} \leq t_{i}-u_{i} \\
& \sum_{j=1}^{n}\left(s_{i j}+r_{i j}\right) x_{j} \leq t_{i}+v_{i} \quad(1 \leq i \leq m) \\
& x_{j} \geq 0 . \quad(1 \leq j \leq n)
\end{aligned}
$$

Since

$$
\max \tilde{z_{i}}=\sum_{j=1}^{n}\left(c^{c}, c^{l}, c^{u}\right)_{i j} x_{j} \quad i=1,2, \ldots, k
$$

we get $\tilde{z}_{i}$ as $\left(z_{i}^{1}, z_{i}^{2}, z_{i}^{3}\right)$ where,

$$
\begin{aligned}
z_{i}{ }^{1} & =\sum_{j=1}^{n}\left(c^{c}\right)_{i j} x_{j}, & i=1,2, \ldots, k \\
z_{i}{ }^{2} & =\sum_{j=1}^{n}\left(c^{c}-c^{l}\right)_{i j} x_{j}, & i=1,2, \ldots, k \\
z_{i}{ }^{3} & =\sum_{j=1}^{n}\left(c^{c}+c^{u}\right)_{i j} x_{j}, & i=1,2, \ldots, k .
\end{aligned}
$$

Thus the objective function of the problem (17) becomes

$$
\max \left(z_{1}^{1}, z_{2}^{2}, z_{3}^{3}, \ldots, z_{k}^{1}, z_{k}^{2}, z_{k}^{3}\right)
$$

To solve this multi-objective problem we use Pareto's method to form weighted objective function

$$
\max w_{1} z_{1}^{1}+w_{2} z_{1}^{2}+w_{3} z_{1}^{3}+\ldots+w_{3 k} z_{k}^{3}
$$

and with the same constraints as in (17) along with the additional constraint $\sum w_{k}=S$. [13]

\section{NUMERICAL EXAMPLE}

In this section, we present a simple example to illustrate the above concepts.

Example. A Company would like to produce two products $A, B$. There are 150 man-hour and $120 \mathrm{~kg}$ material available. In addition, suppose the gained profit from producing each unit of $A$ and $B$ is almost known. The aim of the company is to maximize the overall profit of producing these two products.

Suppose $x_{1}$ is the production amount for the product $A$, and $x_{2}$ is the production amount for the product $B$.

So, this problem can be modeled as:

$$
\begin{aligned}
& \max \tilde{z}=f\left(x_{1}, x_{2}\right)=\tilde{c_{1}} x_{1}+\tilde{c_{2}} x_{2} \\
& \text { s.t. } \\
& (5,2,1) x_{1}+(4,3,1) x_{2} \leq(150,50,40) \\
& (3,2,1) x_{1}+(4,2,1) x_{2} \leq(120,40,30) \\
& \quad x_{1}, x_{2} \geq 0
\end{aligned}
$$

The membership function is:

$$
\mu_{\tilde{c}_{1}}(x)=\left\{\begin{array}{cc}
\frac{x-40}{5}, & 40 \leq x<45 \\
\frac{60-x}{15}, & 45 \leq x<60 \\
0, & \text { o.w. }
\end{array}\right.
$$




$$
\mu_{\tilde{c}_{2}}(x)=\left\{\begin{array}{cc}
\frac{x-30}{6}, & 30 \leq x<36 \\
\frac{40-x}{4}, & 36 \leq x<40 \\
0, & \text { o.w. }
\end{array}\right.
$$

Therefore, the objective function will be as follows:

$$
\max \quad \tilde{z}=f\left(x_{1}, x_{2}\right)=(40,45,60) x_{1}+(30,36,40) x_{2}
$$

According to the discussed algorithm, the human resources constraint can be written as:

$$
\left\{\begin{array}{l}
5 x_{1}+4 x_{2} \leq 150 \\
3 x_{1}+1 x_{2} \leq 100 \\
6 x_{1}+5 x_{2} \leq 190
\end{array}\right.
$$

Also, the constraint for the available materials can be cast as:

$$
\left\{\begin{array}{c}
3 x_{1}+4 x_{2} \leq 120 \\
1 x_{1}+2 x_{2} \leq 80 \\
4 x_{1}+5 x_{2} \leq 150
\end{array}\right.
$$

Now, we can rewrite the above problem as a linear programming:

$$
\begin{aligned}
& \max z=40 x_{1}+30 x_{2} \\
& +45 x_{1}+36 x_{2} \\
& +60 x_{1}+40 x_{2} \\
& \text { s.t. } \\
& 5 x_{1}+4 x_{2} \leq 150 \\
& 3 x_{1}+1 x_{2} \leq 100 \\
& 6 x_{1}+5 x_{2} \leq 190 \\
& 3 x_{1}+4 x_{2} \leq 120 \\
& 1 x_{1}+2 x_{2} \leq 80 \\
& 4 x_{1}+5 x_{2} \leq 150 \\
& x_{1} \geq 0 \\
& x_{2} \geq 0
\end{aligned}
$$

After solving the above problem using LINGO, the following optimal Pareto solution is achieved:

$$
\left(x_{1}^{*}, x_{2}^{*}\right)=(30,0)
$$

Therefore, the above solution is a non-dominated. Here, for solving the linear programming, we maximize the objective-weighted form of the problem as follows:

$$
\begin{aligned}
& \max z\left(x_{1}, x_{2}\right)=w_{1}\left(40 x_{1}+30 x_{2}\right) \\
& +w_{2}\left(45 x_{1}+36 x_{2}\right) \\
& +w_{3}\left(60 x_{1}+40 x_{2}\right)
\end{aligned}
$$

The optimal solution of the above problem can be found using different weights. Note that $\sum_{i=1}^{3} w_{i}=1$.

Table 1. The Optimal Solution of Example

\begin{tabular}{|c|c|c|c|}
\hline $\begin{array}{c}\text { Optimal } \\
\text { Solution }\end{array}$ & $\mathrm{w}_{1}$ & $\mathrm{w}_{2}$ & $\mathrm{w}_{3}$ \\
\hline$(30,0)$ & 0 & 0.5 & 0.5 \\
\hline$(30,0)$ & 0.5 & 0 & 0.5 \\
\hline$(30,0)$ & 0.5 & 0.5 & 0 \\
\hline
\end{tabular}

The optimal solution will be presented as:

$$
\left(x_{1}^{*}, x_{2}^{*}\right)=(30,0)
$$

Therefore,

$$
\begin{gathered}
\max z=f\left(x_{1}^{*}, x_{2}^{*}\right)=f(30,0)=30 \tilde{c}_{1}+0 \tilde{c}_{2} \\
\mu_{f}(x)=\left\{\begin{array}{cc}
\frac{x-1200}{150}, & 1200 \leq x<1350 \\
\frac{1800-x}{450}, & 1350 \leq x<1800 \\
0, & \text { o.w. }
\end{array}\right.
\end{gathered}
$$

\section{CONCLUSION}

In this paper, the linear programming problems with fuzzy budget and constraints have been discussed. An algorith $m$ to solve such problems has been discussed and for more illustration the mentioned algorithm has been applied to a numerical example. As a result of this example, it has been highlighted that the solution is independent from the chosen weights.

\section{REFERENCES}

[1] Bellman, R.E. and Zadeh, L.A., Decision making in fuzzy environment. Management Science. 1970, 17: 141-164.

[2] Dash ,R. B. and Dash, P.D.P., Solving Fuzzy Integer Programming Problem as Multi objective Integer Programming Problem, International Journal of Fuzzy Mathematics and Systems. 2012, 2: 307-314

[3] Delgado M., Verdegay J L., Vila M.A., A general model for fuzzy linear programming. Fuzzy Sets and Systems. 1989, 29: 21-29

[4] Erfanian, H.R., Noori Eskandari, M.H., Vahidian Kamyad A., Solving a Class of Separated Continuous Programming Problems Using Linearization and Discretization, Int. J. Sensing, Computing \& Control, 2011, 1: 117-124.

[5] Erfanian, H.R., Noori Eskandari, M.H., Vahidian Kamyad A., A New Approach for the Generalized First Derivative and Extension It to the Generalized Second Derivative of 
Nonsmooth Functions, I.J. Intelligent Systems and Applications, 2013, 04, 100-107.

[6] Hashem, H.A., Converting Linear Programming Problem with Fuzzy Coefficients into Multi Objective Linear Programming Problem, Australian Journal of Basic and Applied Sciences. 2013, 7(7): 185-189.

[7] Kumar, A., et al. Applied Mathematical Modelling. 2011, 35:817-823

[8] Li G, Guo R. Comments on formulation of fuzzy linear programming problems as four objective constrained optimization problems. Applied Mathematics and Computation. 2007, 186: 941-944

[9] Nasseri, S. H., Behman esh, E., Linear Programming with Triangular Fuzzy Numbers-A Case Study in a Finance and Credit Institute, Fuzzy Inf. Eng. 2013, 3: 295-315

[10] Negoita, C.V., Fuzziness in management OPSA/TIMS Miami. 1970.

[11] Noori Eskandari, M.H., Erfanian, H.R., Vahidian Kamy ad A., Generalized Derivative of Fuzzy Nonsmooth Functions, Journal of Uncertain Sy stems, 2012, 6 (3):214222.

[12] Noori Eskandari, M.H., Erfanian, H.R., Vahidian Kamyad A., Farahi,M.H., Solving a Class of Non-Smooth Optimal Control Problems, I.J. Intelligent Systems and Applications, 2013, 07, 16-22.

[13] Pandit, P.k., Portfolio optimization using fuzzy linear programming, AIP Conference Proceedings. 2013, 1557, 206

[14] Ramik J, Rimanek J. Inequality relation between fuzzy numbers and its use in fuzzy optimization. Fuzzy Sets and Systems. 1985, 16: 123-138

[15] Takeshi, I., Hiroaki, I. and Teruaki, N., A model of crop planning under uncertainty in Agricultural management, Int. J. of Production Economics. 1991, 1: 159-171.

[16] Tanaka, H., Ichihashi, H. and Asai K., A formulation of fuzzy linear programming problem based on comparison of fuzzy numbers, Control and Cybernetics. 1991, 3(3): 185-194.

[17] Thakre, P.A., Shelar D.S. and Thakre, S.P., Solving fuzzy linear programming problem as multi objective linear programming problem, Proceedings of the World Congress on Engineering WCE, London, U.K. 2009.

[18] Zhang, G., Y.H. Wu, M. Remias and J. Lu. Formulation of fuzzy linear programming problems as four-objective constrained optimization problems, Applied Mathematics and Computation. 2003, 139: 383-399.

[19] Zimmermann, H.J., Description and optimization of fuzzy systems, International Journal of General Systems. 1976, 2(4): 209-215.

[20] Zimmermann, H.J., Fuzzy programming and linear programming with several objective functions, Fuzzy Sets and Sy stems. 1978, 1: 45-55.

\section{Authors' Profiles}

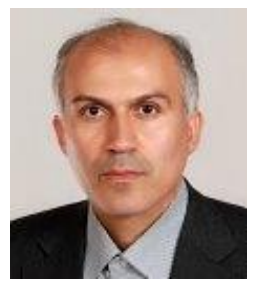

Dr. Hamid Reza Erfanian, Ph.D. in control and optimization from faculty of mathematical sciences, Ferdowsi University of Mashhad, Iran. $\mathrm{He}$ is professor at the faculty of mathematical sciences, University of Science and Culture, Tehran-Iran and his research interests are mainly on optimal control, optimization and financial mathematics.

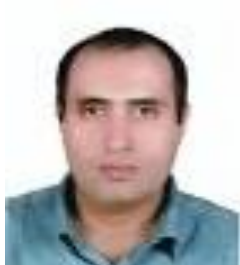

Dr. Mohammad Javad Abdi received his $\mathrm{PhD}$ and MSc in Applied Mathematics from University of Birmingham in England, and Sharif University of Technologv in Iran. respectivelv. His research interests include Numerical Algorithms in Optimization, Portfolio Optimization Problems, and Cardinality and Rank Minimization Problems.

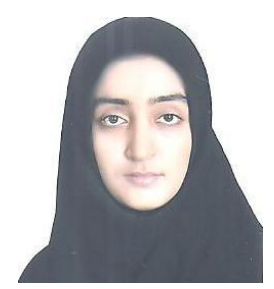

Sahar Kahrizi was born in Iran in 1987. She received her bachelor degree in the field of Applied mathematics at Islamic Azad University Kermanshah Branch in 2010. She is a master student in the field of financial mathemathics at University of Science and Culture.

How to cite this paper: Hamid Reza Erfanian, Mohammad Javad Abdi, Sahar Kahrizi, "Solving a Linear Programming with Fuzzy Constraint and Objective Coefficients", International Journal of Intelligent Systems and Applications (IJISA), Vol.8, No.7, pp.65-72, 2016. DOI: 10.5815/ijisa.2016.07.07 